\title{
A universal magnification theorem. III. Caustics beyond codimension 5
}

\author{
A. B. Aazami ${ }^{1, a)}$ and A. O. Petters ${ }^{1,2, b)}$ \\ ${ }^{1}$ Department of Mathematics, Duke University, Science Drive, Durham, North Carolina \\ 27708, USA \\ ${ }^{2}$ Department of Physics, Duke University, Science Drive, Durham, North Carolina 27708, \\ USA
}

(Received 28 September 2009; accepted 10 November 2009; published online 1 February 2010)

\begin{abstract}
In the final paper of this series, we extend our results on magnification invariants to the infinite family of $A_{n}(n \geq 2), D_{n}(n \geq 4), E_{6}, E_{7}, E_{8}$ caustic singularities. We prove that for families of general mappings between planes exhibiting any caustic singularity of the $A_{n}(n \geq 2), D_{n}(n \geq 4), E_{6}, E_{7}, E_{8}$ family, and for a point in the target space lying anywhere in the region giving rise to the maximum number of lensed images (real preimages), the total signed magnification of the lensed images will always sum to zero. The proof is algebraic in nature and relies on the Euler trace formula. (C) 2010 American Institute of Physics. [doi:10.1063/1.3271043]
\end{abstract}

\section{INTRODUCTION}

In Papers I and II of this series, ${ }^{1,2}$ we established a universal magnification theorem for all higher-order caustics up to codimension 5. It was shown that to each such caustic singularity is associated a magnification sum rule of the form

$$
\sum_{i} \mathfrak{M}_{i}=0
$$

All of these bifurcation sets or big caustics occur in an $n$-parameter space. Slices of the big caustics give rise to caustic metamorphoses that occur in gravitational lensing (see, e.g., Refs. 5, 13, and 11 and Chaps. 7 and 9 in Ref. 12). It was discussed in Paper I, using the hyperbolic umbilic, in particular, how the above magnification relations may be used for substructure studies of four-image lens galaxies.

We now extend these results to all higher-order caustic singularities beyond codimension 5 . These are classified according to Arnol'd's $A, D, E$ classification of Lagrangian map-germs. ${ }^{3}$ Thus, we show that for families of general mappings between planes exhibiting any caustic singularity with corresponding Coxeter-Dynkin diagram of type $A_{n}(n \geq 2), D_{n}(n \geq 4), E_{6}, E_{7}, E_{8}$, and for a point anywhere in the region of the target space giving rise to the maximum number of lensed images (real preimages), the total signed magnification is identically zero. Our proof is algebraic and relies on the Euler trace formula. Finally, we emphasize that the magnification sum relations are geometric invariants, being the reciprocals of Gaussian curvatures at critical points.

The outline of this paper is as follows. In Sec. II we give a brief overview of the $A, D, E$ family of caustic singularities. In Sec. III we state our main theorem. The proof itself is presented in the Appendix.

\footnotetext{
${ }^{\text {a) }}$ Electronic mail: aazami@math.duke.edu.

${ }^{b)}$ Electronic mail: petters@math.duke.edu.
} 
TABLE I. For each type of Coxeter-Dynkin diagram listed, indexed by $n$, the second column shows the corresponding universal local forms of the smooth $(n-1)$-parameter family of general functions $F_{\mathbf{c}, \mathbf{s}}$, along with their $(n-3)$-parameter family of induced general maps $\mathbf{f}_{\mathbf{c}}$ between planes. This classification is due to Arnol'd (Ref. 3).

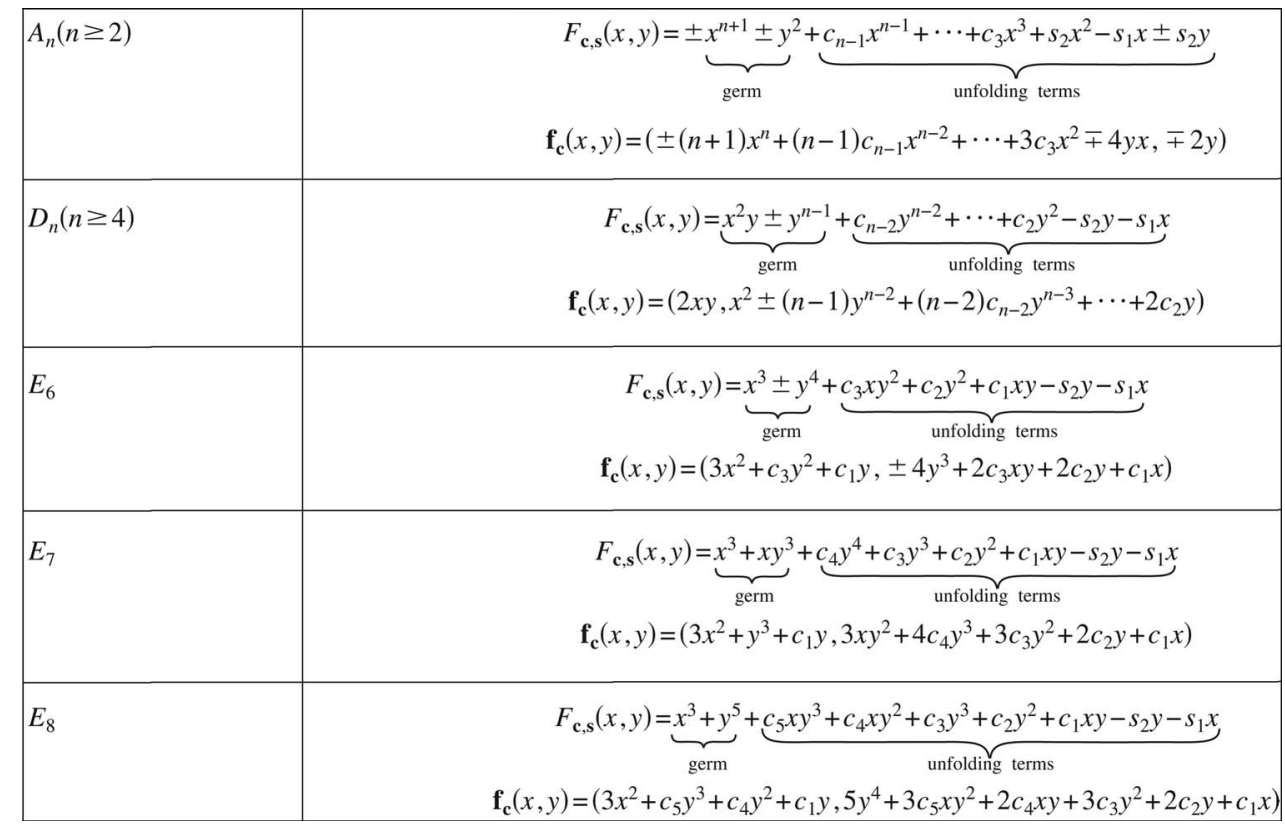

\section{HIGHER-ORDER CAUSTICS OF THE $A, D, E$ FAMILY}

We inherit the notation and terminology of Paper II. To that end, consider a smooth $n$-parameter family $F_{\mathbf{c}, \mathbf{s}}(\mathbf{x})$ of functions on an open subset of $\mathbb{R}^{2}$ that induces a smooth $(n-2)$-parameter family of mappings $\mathbf{f}_{\mathbf{c}}(\mathbf{x})$ between planes $(n \geq 2)$. One uses $F_{\mathbf{c}, \mathbf{s}}$ to construct a Lagrangian submanifold that is projected into the space $\{\mathbf{c}, \mathbf{s}\}=\mathbb{R}^{n-2} \times \mathbb{R}^{2}$. The caustics of $\mathbf{f}_{\mathbf{c}}$ will then be the critical values of the projection (see, e.g., Refs. 9, 10, and 7 and pp. 276-286 in Ref. 12). These projections are called Lagrangian maps, and they are differentiably equivalent to $\mathbf{f}_{\mathbf{c}}$.

Arnol'd classified all stable simple Lagrangian map-germs of $n$-dimensional Lagrangian submanifolds by their generating family $F_{\mathbf{c}, \mathbf{s}}$ (Ref. 3, pp. 330 and 331 in Ref. 4, and p. 282 in Ref. 12). In the process, he found a connection between his classification and the Coxeter-Dynkin diagrams of the simple Lie algebras of types $A_{n}(n \geq 2), D_{n}(n \geq 4), E_{6}, E_{7}, E_{8}$. This classification is shown in Table I. The singularities in Paper II arose as follows: $A_{2}$ (fold), $A_{3}$ (cusp), $D_{4}^{-}$(elliptic umbilic), $D_{4}^{+}$(hyperbolic umbilic), $A_{4}$ (swallowtail), $A_{5}$ (butterfly), $D_{5}$ (parabolic umbilic), $A_{6}$ (wigwam), $E_{6}$ (symbolic umbilic), $D_{6}^{-}$(second elliptic umbilic), and $D_{6}^{+}$(second hyperbolic umbilic).

For the $\mathbf{f}_{\mathbf{c}}$ shown in Table I, call $\mathbf{x} \in \mathbb{R}^{2}$ a lensed image (or a real preimage) of the target point $\mathbf{s} \in \mathbb{R}^{2}$ if $\mathbf{f}_{\mathbf{c}}(\mathbf{x})=\mathbf{s}$ (in particular, our lensed images are always in $\mathbb{R}^{2}$, not $C^{2}$ ). A point $\mathbf{x}_{i} \in \mathbb{R}^{2}$ is a lensed image of the target point $\mathbf{s} \in \mathbb{R}^{2}$ if and only if $\mathbf{x}_{i}$ is a critical point of $F_{\mathbf{c}, \mathbf{s}}$ (relative to a gradient in $\mathbf{x})$. Next, we define the magnification $\mathfrak{M}\left(\mathbf{x}_{i} ; \mathbf{s}\right)$ at a critical point $\mathbf{x}_{i}$ of the family $F_{\mathbf{c}, \mathbf{s}}$ by the reciprocal of the Gaussian curvature at the point $\left(\mathbf{x}_{i}, F_{\mathbf{c}, \mathbf{s}}\left(\mathbf{x}_{i}\right)\right)$ in the graph of $F_{\mathbf{c}, \mathbf{s}}$,

$$
\mathfrak{M}\left(\mathbf{x}_{i} ; \mathbf{s}\right)=\frac{1}{\operatorname{Gauss}\left(\mathbf{x}_{i}, F_{\mathbf{c}, \mathbf{s}}\left(\mathbf{x}_{i}\right)\right)} .
$$

This makes it clear that the magnification invariants established in our theorem are geometric invariants. In addition, since Gauss $\left(\mathbf{x}_{i}, F_{\mathbf{c}, \mathbf{s}}\left(\mathbf{x}_{i}\right)\right)=\operatorname{det}\left(\operatorname{Hess} F_{\mathbf{c}, \mathbf{s}}\right)\left(\mathbf{x}_{i}\right)$, and since each $\mathbf{f}_{\mathbf{c}}$ in Table I satisfies $\operatorname{det}\left(\operatorname{Jac} \mathbf{f}_{\mathbf{c}}\right)=\operatorname{det}\left(\right.$ Hess $\left.F_{\mathbf{c}, \mathbf{s}}\right)$, we can also express the magnification in terms of $\mathbf{f}_{\mathbf{c}}$, 


$$
\mathfrak{M}\left(\mathbf{x}_{i} ; \mathbf{s}\right)=\frac{1}{\operatorname{det}\left(\operatorname{Jac} \mathbf{f}_{\mathbf{c}}\right)\left(\mathbf{x}_{i}\right)},
$$

where $\mathbf{x}_{i}$ is a lensed image of $\mathbf{s}$ under $\mathbf{f}_{\mathbf{c}}$. We now proceed to our main theorem.

\section{STATEMENT OF THE MAIN THEOREM}

Theorem 1: For any of the universal, smooth (n-1)-parameter family of general functions $F_{\mathbf{c}, \mathbf{s}}$ (or induced general mappings $\mathbf{f}_{\mathbf{c}}$ ) in Table $I$, and for any noncaustic point $\mathbf{s}$ (light source position) in the indicated region, the following results hold for the magnification $\mathfrak{M}_{i} \equiv \mathfrak{M}\left(\mathbf{x}_{i} ; \mathbf{s}\right)$ :

(1) $A_{n}(n \geq 2)$ obeys the magnification relation in the $n$-image region: $\sum_{i=1}^{n} \mathfrak{M}_{i}=0$,

(2) $D_{n}(n \geq 4)$ obeys the magnification relation in the $n$-image region: $\sum_{i=1}^{n} \mathfrak{M}_{i}=0$,

(3) $E_{6}$ obeys the magnification relation in the six-image region: $\sum_{i=1}^{6} \mathfrak{M}_{i}=0$,

(4) $E_{7}$ obeys the magnification relation in the seven-image region: $\sum_{i=1}^{7} \mathfrak{M}_{i}=0$,

(5) $E_{8}$ obeys the magnification relation in the eight-image region: $\sum_{i=1}^{8} \mathfrak{M}_{i}=0$.

The magnification relations for $A_{2}$ (fold) and $A_{3}$ (cusp) are known. ${ }^{6,12-15}$ Those for $D_{4}^{-}$(elliptic umbilic), $D_{4}^{+}$(hyperbolic umbilic), $A_{4}$ (swallowtail), $A_{5}$ (butterfly), $D_{5}$ (parabolic umbilic), $A_{6}$ (wigwam), $E_{6}$ (symbolic umbilic), $D_{6}^{-}$(second elliptic umbilic), and $D_{6}^{+}$(second hyperbolic umbilic) were discovered recently in Refs. 1 and 2.

Remarks: First, the results of Theorem 1 actually apply even when the noncaustic point $\mathbf{s}$ is not in the maximum number of real preimage region. However, preimages from $\mathrm{C}^{2}$ will appear, which are unphysical in gravitational lensing. Second, it is important to point out that Theorem 1 is not a direct consequence of the Euler-Jacobi formula, of multidimensional residue integral methods, or of Lefschetz fixed point theory because some of the singularities have fixed points at infinity that must be treated individually. Third, we point out that for $n \geq 6$ there are Lagrangian maps that cannot be approximated by stable Lagrangian map-germs. ${ }^{3}$

\section{CONCLUSION}

This paper presented a theorem about the magnification of lensed images for all caustic singularities appearing in the infinite family of $A_{n}(n \geq 2), D_{n}(n \geq 4), E_{6}, E_{7}, E_{8}$ caustic singularities. We proved that for families of general mappings between planes locally exhibiting any caustic singularity of the $A_{n}(n \geq 2), D_{n}(n \geq 4), E_{6}, E_{7}, E_{8}$ family, and for a target point lying anywhere in the region giving rise to the maximum number of lensed images (real preimages), the total signed magnification of the lensed images will always sum to zero. The signed magnifications are geometric invariants as they are Gaussian curvatures at critical points. The proof was algebraic in nature and made use of the Euler trace formula. Our result goes beyond previous work that considered singularities up to codimension five.

\section{ACKNOWLEDGMENTS}

A.O.P. acknowledges the support of the NSF under Grant No. DMS-0707003. Part of this work was conducted at the Petters Research Institute, Belize.

\section{APPENDIX: PROOF OF THE MAIN THEOREM}

\section{Overview of the method and the Euler trace formula}

We summarize the key elements of our algebraic method (see Paper II for a detailed presentation). Consider any polynomial $\varphi(x)=a_{n} x^{n}+\cdots+a_{1} x+a_{0} \in \mathrm{C}[x]$ with distinct roots $x_{1}, \ldots, x_{n}$ and any rational function $h(x) \in R$, where $R \subset \mathrm{C}(x)$ is the subring of rational functions defined at the roots of $\varphi(x)$. In Paper II we showed algebraically that for any rational function $h(x) \in R$, the following holds: 


$$
\sum_{i=1}^{n} h\left(x_{i}\right)=\frac{b_{n-1}}{a_{n}} \quad(\text { Euler trace formula })
$$

where $b_{n-1}$ is the $(n-1)$ st coefficient of the unique polynomial representative $r(x)$ of degree less than $n$ in the coset $\overline{\varphi^{\prime}(x) h(x)} \in R /(\varphi(x)$ ). (An alternate proof of the Euler trace formula using residues can be found in Ref. 8.) We employ the Euler trace formula as follows. For any caustic singularity in Table I, we shall realize the lensed images of its corresponding family of mappings $\mathbf{f}_{\mathbf{c}}$ as solutions of a polynomial in one variable, obtained by eliminating one of the preimage coordinates, say $y$. Denote this polynomial by

$$
\varphi(x)=a_{n} x^{n}+\cdots+a_{1} x+a_{0} \in \mathbb{C}[x] .
$$

Generically, we can assume that the roots of $\varphi(x)$ are distinct, an assumption made throughout the paper. We would then be able to express the magnification $\mathfrak{M}(x, y ; \mathbf{s})$ at a general preimage point $(x, y)$ as a function of one variable, in this case $x$, so that

$$
\mathfrak{M}(x, y(x) ; \mathbf{s})=\frac{1}{\operatorname{det}\left(\operatorname{Jac} \mathbf{f}_{\mathbf{c}}\right)(x, y(x))} \equiv \frac{1}{\operatorname{det}\left(\operatorname{Jac} \mathbf{f}_{\mathbf{c}}\right)(x)} \equiv \mathfrak{M}(x),
$$

where the explicit notational dependence on $\mathbf{s}$ is dropped for simplicity. Since we shall consider only noncaustic target points $\mathbf{s}$ giving rise to lensed images $\left(x_{i}, y_{i}\left(x_{i}\right)\right)$, we have $\operatorname{det}\left(\operatorname{Jac} \mathbf{f}_{\mathbf{c}}\right)\left(x_{i}\right)$ $\neq 0$. We thus know that for the singularities in Table $\mathrm{I}$, the rational function $\mathfrak{M}(x)$ is defined at the roots of $\varphi(x)$, i.e., $\mathfrak{M}(x) \in R$. Now, denote by $\mathfrak{m}(x)$ the unique polynomial representative of degree less than $n$ in the coset $\overline{\varphi^{\prime}(x) \mathfrak{M}(x)} \in R /(\varphi(x))$, and let $b_{n-1}$ be its $(n-1)$ st coefficient. In the notation used above, we have $h(x) \equiv \mathfrak{M}(x)$ and $r(x) \equiv \mathfrak{m}(x)$. Euler's trace formula (Corollary A1) then tells us immediately that the total signed magnification satisfies

$$
\sum_{i} \mathfrak{M}_{i}=\frac{b_{n-1}}{a_{n}}
$$

It therefore remains to determine the coefficient $b_{n-1}$ for each caustic singularity in Table I.

\section{Magnification sum rule for type $A_{n}$}

We begin with type $A_{n}, n \geq 2$. Since the cases $2 \leq n \leq 6$ were treated in Paper II, we will consider $n \geq 7$ here. The $(n-1)$-parameter family of general functions $F^{A_{n}}$ is given in Ref. 3 by

$$
F^{A_{n}}(x, y)=\underbrace{ \pm x^{n+1} \pm y^{2}}_{\text {germ }}+\underbrace{c_{n-1} x^{n-1}+c_{n-2} x^{n-2}+\cdots+c_{3} x^{3}+c_{2} x^{2}+c_{1} x}_{\text {unfolding terms }} .
$$

To convert this into the form shown in Table I, we use the following coordinate transformation on the domain $\{(x, y)\}=\mathbb{R}^{2}$ :

$$
(x, y) \mapsto\left(x, y+\frac{c_{2}}{2}\right) .
$$

This transforms Eq. (A3) to

$$
F_{\mathbf{c}, \mathbf{s}}^{A_{n}}(x, y)= \pm x^{n+1} \pm y^{2}+c_{n-1} x^{n-1}+c_{n-2} x^{n-2}+\cdots+c_{3} x^{3}+s_{2} x^{2}-s_{1} x \pm s_{2} y,
$$

where $c_{1} \equiv-s_{1}$ and $c_{2} \equiv s_{2}$. The parameters $s_{1}, s_{2}$ are to be interpreted in the context of gravitational lensing as the rectangular coordinates on the source plane $S=\mathbb{R}^{2}$. Note that we omitted the constant term from Eq. (A5) since it will not affect any of our results below. Note also that 


$$
\operatorname{det}\left(\operatorname{Hess} F^{A_{n}}\right)=\operatorname{det}\left(\operatorname{Hess} F_{\mathbf{c}, \mathbf{s}}^{A_{n}}\right) \text {, }
$$

so that the magnification [as defined in Eq. (1)] is unaltered. We will work with the form of $F_{\mathbf{c}, \mathbf{s}}^{A_{n}}$ in Eq. (A5). The corresponding (n-3)-parameter family of general mappings $\mathbf{f}_{\mathbf{c}}^{A_{n}}: \mathbb{R}^{2} \rightarrow \mathbb{R}^{2}$ is

$$
\mathbf{f}_{\mathbf{c}}^{A_{n}}(x, y)=\left( \pm(n+1) x^{n}+(n-1) c_{n-1} x^{n-2}+(n-2) c_{n-2} x^{n-3}+\cdots+3 c_{3} x^{2} \mp 4 y x, \mp 2 y\right)=\left(s_{1}, s_{2}\right) .
$$

Here, $\mathbf{s}=\left(s_{1}, s_{2}\right)$ is a noncaustic target point lying in the region with the maximum number of lensed images. Since $s_{2}= \pm 2 y$, we can eliminate $y$ to obtain a polynomial in the variable $x$,

$$
\varphi_{A_{n}}(x) \equiv \pm(n+1) x^{n}+(n-1) c_{n-1} x^{n-2}+(n-2) c_{n-2} x^{n-3}+\cdots+3 c_{3} x^{2}+2 s_{2} x-s_{1},
$$

whose $n$ roots are the $x$-coordinates of the lensed images $\mathbf{x}_{i}$ of $\mathbf{s}$. The Jacobian determinant of $\mathbf{f}_{\mathbf{c}}^{A_{n}}$ expressed in the single variable $x$ is

$$
\begin{aligned}
\operatorname{det}\left(\operatorname{Jac} \mathbf{f}_{\mathbf{c}}^{A_{n}}\right)(x)= & \mp 2\left[ \pm n(n+1) x^{n-1}+(n-2)(n-1) c_{n-1} x^{n-3}+(n-3)(n-2) c_{n-2} x^{n-4}+\cdots+6 c_{3} x\right. \\
& \left.+2 s_{2}\right] .
\end{aligned}
$$

A comparison of Eqs. (A6) and (A7) then shows that

$$
\pm 2 \varphi_{A_{n}}^{\prime}(x)=\operatorname{det}\left(\operatorname{Jac} \mathbf{f}_{\mathbf{c}}^{A_{n}}\right)(x)=\frac{1}{\mathfrak{M}(x)}
$$

We thus have

$$
\varphi_{A_{n}}^{\prime}(x) \mathfrak{M}(x)= \pm \frac{1}{2} .
$$

Thus, the unique polynomial representative of the coset $\overline{\varphi_{A_{n}}^{\prime}(x) \mathfrak{M}(x)}$ is the polynomial $\mathfrak{m}(x)$ $\equiv \pm 1 / 2$, whose $(n-1)$ st coefficient is $b_{n-1}=0$ for all $n \geq 7$. Euler's trace formula in the form of Eq. (A2) then tells us that the total signed magnification is

$$
\sum_{i=1}^{n} \mathfrak{M}_{i}=0 \quad\left(A_{n}, n \geq 2\right)
$$

\section{Magnification sum rules for type $D_{n}$}

For type $D_{n}, n \geq 4$, the cases $4 \leq n \leq 6$ were treated in Paper II, so we will consider $n \geq 7$ here. The corresponding (n-3)-parameter family of induced general maps $\mathbf{f}_{\mathbf{c}}^{D_{n}^{ \pm}} \mathbb{R}^{2} \rightarrow \mathbb{R}^{2}$ is shown in Table I,

$$
\mathbf{f}_{\mathbf{c}}^{D_{n}^{ \pm}}(x, y)=\left(2 x y, x^{2} \pm(n-1) y^{n-2}+(n-2) c_{n-2} y^{n-3}+\cdots+(n-i) c_{n-i} y^{n-(i+1)}+\cdots+2 c_{2} y\right)=\left(s_{1}, s_{2}\right) .
$$

Once again the point $\mathbf{s}=\left(s_{1}, s_{2}\right)$ is a noncaustic target point lying in the region with the maximum number of lensed images. This time, however, we eliminate $x$ to obtain a polynomial in the variable $y$,

$$
\varphi_{D_{n}^{ \pm}}(y) \equiv \pm 4(n-1) y^{n}+4(n-2) c_{n-2} y^{n-1}+\cdots+4(n-i) c_{n-i} y^{n-(i-1)}+\cdots+8 c_{2} y^{3}-4 s_{2} y^{2}+s_{1}^{2},
$$

whose $n$ roots are the $y$-coordinates of the $n$ lensed images $\mathbf{x}_{i}$ of $\mathbf{s}$. The derivative of $\varphi_{D_{n}^{ \pm}}(y)$ is 


$$
\begin{aligned}
\varphi_{D_{n}^{ \pm}}^{\prime}(y)= & \pm 4 n(n-1) y^{n-1}+4(n-1)(n-2) c_{n-2} y^{n-2}+\cdots+4(n-(i-1))(n-i) c_{n-i} y^{n-i}+\cdots \\
& +24 c_{2} y^{2}-8 s_{2} y,
\end{aligned}
$$

while the Jacobian determinant of $\mathbf{f}_{\mathbf{c}}^{D_{n}^{ \pm}}$is

$$
\begin{aligned}
\operatorname{det}\left(\operatorname{Jac} \mathbf{f}_{\mathbf{c}}^{D_{n}^{ \pm}}\right)(x, y)= & \operatorname{det}\left[\begin{array}{ll}
2 y & 2 x \\
2 x & \pm(n-2)(n-1) y^{n-3}+(n-3)(n-2) c_{n-2} y^{n-4}+\cdots+2 c_{2}
\end{array}\right] \\
= & \pm 2(n-2)(n-1) y^{n-2}+2(n-3)(n-2) c_{n-2} y^{n-3}+\cdots \cdots+2(n-(i+1))(n \\
& -i) c_{n-i} y^{n-(i+1)}+\cdots+4 c_{2} y-4 x^{2} .
\end{aligned}
$$

We can use Eq. (A8) to eliminate $x$ as follows:

$$
\begin{aligned}
= & \pm 2(n-2)(n-1) y^{n-2}+2(n-3)(n-2) c_{n-2} y^{n-3}+\cdots+2(n-(i+1))(n-i) c_{n-i} y^{n-(i+1)}+\cdots \\
& +4 c_{2} y+4 \underbrace{( \pm(\text { by Eq. (A8)) }}_{=-x^{2}}(n-1) y^{n-2}+(n-2) c_{n-2} y^{n-3}+\cdots+(n-i) c_{n-i} y^{n-(i+1)}+\cdots+2 c_{2} y-s_{2}) \\
= & \pm 2 n(n-1) y^{n-2}+2(n-1)(n-2) c_{n-2} y^{n-3}+\cdots+2(n-(i-1))(n-i) c_{n-i} y^{n-(i+1)}+\cdots+12 c_{2} y \\
& -4 s_{2}=\operatorname{det}\left(\operatorname{Jac} \mathbf{f}_{\mathbf{c}}^{D_{n}^{ \pm}}\right)(y)=\mathfrak{M}(y)^{-1} .
\end{aligned}
$$

A comparison with Eq. (A9) then shows that

$$
\varphi_{D_{n}^{ \pm}}^{\prime}(y) \mathfrak{M}(y)=2 y .
$$

The unique polynomial representative of the coset $\overline{\varphi_{D_{n}^{ \pm}}^{\prime}(y) \mathfrak{M}(y)}$ is therefore the polynomial $\mathfrak{m}(y) \equiv 2 y$, whose $(n-1)$ st coefficient is $b_{n-1}=0$ for all $n \geq 7$. Equation (A2) then tells us that the total signed magnification is

$$
\sum_{i=1}^{n} \mathfrak{M}_{i}=0 \quad\left(D_{n}, n \geq 4\right) .
$$

\section{Magnification sum rules for types $E_{n}$}

The case $E_{6}$ corresponds to the symbolic umbilic, whose magnification sum rule was proved in Paper II. For type $E_{7}$, Table I gives the corresponding 4-parameter family of induced general $\operatorname{maps} \mathbf{f}_{\mathbf{c}}^{E_{7}}: \mathbb{R}^{2} \rightarrow \mathbb{R}^{2}$

$$
\mathbf{f}_{\mathbf{c}}^{E_{7}}(x, y)=\left(3 x^{2}+y^{3}+c_{1} y, 3 x y^{2}+4 c_{4} y^{3}+3 c_{3} y^{2}+2 c_{2} y+c_{1} x\right)=\left(s_{1}, s_{2}\right) .
$$

Once again, the point $\mathbf{s}=\left(s_{1}, s_{2}\right)$ is a noncaustic point lying in the region with the maximum number of lensed images. We eliminate $x$ to obtain a polynomial in the variable $y$,

$$
\begin{aligned}
\varphi_{E_{7}}(y) \equiv & 9 y^{7}+48 c_{4}^{2} y^{6}+\left(15 c_{1}+72 c_{3} c_{4}\right) y^{5}+\left(27 c_{3}^{2}+48 c_{2} c_{4}-9 s_{1}\right) y^{4}+\left(7 c_{1}^{2}+36 c_{2} c_{3}-24 c_{4} s_{2}\right) y^{3} \\
& +\left(12 c_{2}^{2}-6 c_{1} s_{1}-18 c_{3} s_{2}\right) y^{2}+\left(c_{1}^{3}-12 c_{2} s_{2}\right) y-c_{1}^{2} s_{1}+3 s_{2}^{2},
\end{aligned}
$$

whose seven roots are the $y$-coordinates of the seven lensed images $\mathbf{x}_{i}$ of $\mathbf{s}$. The derivative of $\varphi_{E_{7}}(y)$ is 


$$
\begin{aligned}
\varphi_{E_{7}}^{\prime}(y)= & 63 y^{6}+288 c_{4}^{2} y^{5}+\left(75 c_{1}+360 c_{3} c_{4}\right) y^{4}+\left(108 c_{3}^{2}+192 c_{2} c_{4}-36 s_{1}\right) y^{3}+\left(21 c_{1}^{2}+108 c_{2} c_{3}\right. \\
& \left.-72 c_{4} s_{2}\right) y^{2}+\left(24 c_{2}^{2}-12 c_{1} s_{1}-36 c_{3} s_{2}\right) y+c_{1}^{3}-12 c_{2} s_{2},
\end{aligned}
$$

while the Jacobian determinant of $\mathbf{f}_{\mathbf{c}}^{E_{7}}$ is

$$
\begin{aligned}
\operatorname{det}\left(\operatorname{Jac} \mathbf{f}_{\mathbf{c}}^{\boldsymbol{Z}_{7}}\right)(x, y)= & \operatorname{det}\left[\begin{array}{cc}
6 x & 3 y^{2}+c_{1} \\
3 y^{2}+c_{1} & 6 x y+12 c_{4} y^{2}+6 c_{3} y+2 c_{2}
\end{array}\right]=36 x^{2} y+72 c_{4} x y^{2}+36 c_{3} x y+12 c_{2} x \\
& -9 y^{4}-6 c_{1} y^{2}-c_{1}^{2} .
\end{aligned}
$$

To convert this into a function in the single variable $y$, we use Eq. (A10) twice, as follows:

$$
\begin{aligned}
& =36 x^{2} y+72 c_{4} x y^{2}+36 c_{3} x y+12 c_{2} x-9 y^{4}-6_{1} y^{2}-c_{1}^{2} \\
& =36 y \underbrace{\left(\frac{s_{1}-c_{1} y-y^{3}}{3}\right)}_{=x^{2} \text { (by Eq. (A10)) }}+\left(72 c_{4} y^{2}+36 c_{3} y+12 c_{2}\right)(\underbrace{\left(\frac{s_{2}-4 c_{4} y^{3}-3 c_{3} y^{2}-2 c_{2} y}{3 y^{2}+c_{1}}\right)}_{=x \text { (by Eq. (A10)) }}-9 y^{4}-6 c_{1} y^{2}-c_{1}^{2} \\
& \vdots \\
& =\left(c_{1}+3 y^{2}\right)^{-1}\left[-c_{1}^{3}+12 c_{2} s_{2}+\left(-24 c_{2}^{2}+12 c_{1} s_{1}+36 c_{3} s_{2}\right) y+\left(-21 c_{1}^{2}-108 c_{2} c_{3}+72 c_{4} s_{2}\right) y^{2}\right. \\
& \left.+\left(-108 c_{3}^{2}-192 c_{2} c_{4}+36 s_{1}\right) y^{3}-\left(75 c_{1}+360 c_{3} c_{4}\right) y^{4}-288 c_{4}^{2} y^{5}-63 y^{6}\right] \\
& =\operatorname{det}\left(\operatorname{Jac} \mathbf{f}_{\mathbf{c}}^{E_{7}}\right)(y)=\mathfrak{M}(y)^{-1} .
\end{aligned}
$$

A comparison of Eqs. (A11) and (A12) then shows that

$$
\varphi_{E_{7}}^{\prime}(y) \mathfrak{M}(y)=-\left(c_{1}+3 y^{2}\right),
$$

and so the unique polynomial representative of the coset $\overline{\varphi_{E_{7}}^{\prime}(y) \mathfrak{M}(y)}$ is the polynomial $\mathfrak{m}(y) \equiv$ $-\left(c_{1}+3 y^{2}\right)$, whose sixth coefficient is $b_{6}=0$. We conclude via Eq. (A2) that the total signed magnification is

$$
\sum_{i=1}^{7} \mathfrak{M}_{i}=0 \quad\left(E_{7}\right) .
$$

For type $E_{8}$, Table I gives the corresponding 5-parameter family of induced general maps $\mathbf{f}_{\mathbf{c}}^{E_{8}}: \mathbb{R}^{2} \rightarrow \mathbb{R}^{2}$

$$
\mathbf{f}_{\mathbf{c}}^{E_{8}}(x, y)=\left(3 x^{2}+c_{5} y^{3}+c_{4} y^{2}+c_{1} y, 5 y^{4}+3 c_{5} x y^{2}+2 c_{4} x y+3 c_{3} y^{2}+2 c_{2} y+c_{1} x\right)=\left(s_{1}, s_{2}\right) .
$$

As usual, the point $\mathbf{s}=\left(s_{1}, s_{2}\right)$ is a noncaustic target point lying in the region with the maximum number of lensed images. We eliminate $x$ to obtain a polynomial in the variable $y$,

$$
\begin{aligned}
\varphi_{E_{8}}(y) \equiv & 75 y^{8}+9 c_{5} y^{7}+\left(90 c_{3}+21 c_{4} c_{5}^{2}\right) y^{6}+\left(60 c_{2}+16 c_{4}^{2} c_{5}+15 c_{1} c_{5}^{2}\right) y^{5}+\left(27 c_{3}^{2}+4 c_{4}^{3}+22 c_{1} c_{4} c_{5}\right. \\
& \left.-9 c_{5}^{2} s 1-30 s_{2}\right) y^{4}+\left(36 c_{2} c_{3}+8 c_{1} c_{4}^{2}+7 c_{1}^{2} c_{5}-12 c_{4} c_{5} s_{1}\right) y^{3}+\left(12 c_{2}^{2}+5 c_{1}^{2} c_{4}-4 c_{4}^{2} s_{1}\right. \\
& \left.-6 c_{1} c_{5} s_{1}-18 c_{3} s_{2}\right) y^{2}+\left(c_{1}^{3}-4 c_{1} c_{4} s_{1}-12 c_{2} s_{2}\right) y-c_{1}^{2} s_{1}+3 s_{2}^{2},
\end{aligned}
$$

whose eight roots are the $y$-coordinates of the eight lensed images $\mathbf{x}_{i}$ of $\mathbf{s}$. The derivative of $\varphi_{E_{8}}(y)$ is 


$$
\begin{aligned}
\varphi_{E_{8}}^{\prime}(y)= & 600 y^{7}+63 c_{5} y^{6}+\left(540 c_{3}+126 c_{4} c_{5}^{2}\right) y^{5}+\left(300 c_{2}+80 c_{4}^{2} c_{5}+75 c_{1} c_{5}^{2}\right) y^{4}+\left(108 c_{3}^{2}+16 c_{4}^{3}\right. \\
& \left.+88 c_{1} c_{4} c_{5}-36 c_{5}^{2} s_{1}-120 s_{2}\right) y^{3}+\left(108 c_{2} c_{3}+24 c_{1} c_{4}^{2}+21 c_{1}^{2} c_{5}-36 c_{4} c_{5} s_{1}\right) y^{2}+\left(24 c_{2}^{2}\right. \\
& \left.+10 c_{1}^{2} c_{4}-8 c_{4}^{2} s_{1}-12 c_{1} c_{5} s_{1}-36 c_{3} s_{2}\right) y+c_{1}^{3}-4 c_{1} c_{4} s_{1}-12 c_{2} s_{2},
\end{aligned}
$$

and the Jacobian determinant of $\mathbf{f}_{\mathbf{c}}^{E_{8}}$ is

$$
\begin{aligned}
\operatorname{det}\left(\operatorname{Jac} \mathbf{f}_{\mathbf{c}}^{E_{8}}\right)(x, y)= & \operatorname{det}\left[\begin{array}{cc}
6 x & 3 c_{5} y^{2}+2 c_{4} y+c_{1} \\
3 c_{5} y^{2}+2 c_{4} y+c_{1} & 20 y^{3}+6 c_{5} x y+2 c_{4} x+6 c_{3} y+2 c_{2}
\end{array}\right]=-c_{1}^{2}+12 c_{2} x \\
& +12 c_{4} x^{2}-4 c_{1} c_{4} y+36 c_{3} x y+36 c_{5} x^{2} y-4 c_{4}^{2} y^{2}-6 c_{1} c_{5} y^{2}-12 c_{4} c_{5} y^{3}+120 x y^{3} \\
& -9 c_{5}^{2} y^{4} .
\end{aligned}
$$

Similar to the case $E_{7}$ above, we convert this to a function in the single variable $y$ with the aid of Eq. (A13),

$$
\begin{aligned}
& =-c_{1}^{2}+12 c_{2} x+12 c_{4} x^{2}-4 c_{1} c_{4} y+36 c_{3} x y+36 c_{5} x^{2} y-4 c_{4}^{2} y^{2}-6 c_{1} c_{5} y^{2}-12 c_{4} c_{5} y^{3}+120 x y^{3}-9 c_{5}^{2} y^{4} \\
& =\left(12 c_{2}+36 c_{3} y+120 y^{3}\right) \underbrace{\left(\frac{s_{2}-5 y^{4}-3 c_{3} y^{2}-2 c_{2} y}{3 c_{5} y^{2}+2 c_{4} y+c_{1}}\right)}_{=x \text { (by Eq. (A13)) }}+\left(12 c_{4}+36 c_{5} y\right) \underbrace{\left(\frac{s_{1}-c_{1} y-c_{4} y^{2}-c_{5} y^{3}}{3}\right)}_{=x^{2} \text { (by Eq. (A13)) }} \\
& -c_{1}^{2}-4 c_{1} c_{4} y-4 c_{4}^{2} y^{2}-6 c_{1} c_{5} y^{2}-12 c_{4} c_{5} y^{3}-9 c_{5}^{2} y^{4} \\
& \text { ! } \\
& =\left(c_{1}+2 c_{4} y+3 c_{5} y^{2}\right)^{-1}\left[-c_{1}^{3}+4 c_{1} c_{4} s_{1}+12 c_{2} s_{2}+\left(-24 c_{2}^{2}-10 c_{1}^{2} c_{4}+8 c_{4}^{2} s_{1}+12 c_{1} c_{5} s_{1}+36 c_{3} s_{2}\right) y\right. \\
& +\left(-108 c_{2} c_{3}-24 c_{1} c_{4}^{2}-21 c_{1}^{2} c_{5}+36 c_{4} c_{5} s_{1}\right) y^{2}+\left(-108 c_{3}^{2}-16 c_{4}^{3}-88 c_{1} c_{4} c_{5}+36 c_{5}^{2} s_{1}+120 s_{2}\right) y^{3} \\
& \left.+\left(-300 c_{2}-80 c_{4}^{2} c_{5}-75 c_{1} c_{5}^{2}\right) y^{4}-\left(540 c_{3}+126 c_{4} c_{5}^{2}\right) y^{5}-63 c_{5}^{3} y^{6}-600 y^{7}\right] \\
& =\operatorname{det}\left(\operatorname{Jac} \mathbf{f}_{\mathbf{c}}^{E_{8}}\right)(y)=\mathfrak{M}(y)^{-1} \text {. }
\end{aligned}
$$

A comparison of Eqs. (A14) and (A15) thus shows that

$$
\varphi_{E_{8}}^{\prime}(y) \mathfrak{M}(y)=-\left(c_{1}+2 c_{4} y+3 c_{5} y^{2}\right)
$$

and so the unique polynomial representative of the coset $\overline{\varphi_{E_{8}}^{\prime}(y) \mathfrak{M}(y)}$ is the polynomial $\mathfrak{m}(y) \equiv$ $-\left(c_{1}+2 c_{4} y+3 c_{5} y^{2}\right)$, whose seventh coefficient is $b_{7}=0$. Euler's trace formula tells us once again that

$$
\sum_{i=1}^{8} \mathfrak{M}_{i}=0 \quad\left(E_{8}\right)
$$

This completes the proof of the magnification relations for all higher-order caustic singularities appearing in the infinite $A_{n}(n \geq 2), D_{n}(n \geq 4), E_{6}, E_{7}, E_{8}$ family.

\footnotetext{
${ }^{1}$ Aazami, A. B. and Petters, A. O., J. Math. Phys. 50, 032501 (2009), Paper I.

${ }^{2}$ Aazami, A. B. and Petters, A. O., J. Math. Phys. 50, 082501 (2009), (Paper II).

${ }^{3}$ Arnol'd, V. I., Funct. Anal. Appl. 6, 254 (1973).

${ }^{4}$ Arnol'd, V. I., Gusein-Zade, S. M., and Varchenko, A. N., Singularities of Differentiable Maps (Birkhäuser, Boston, 1985), Vol. 1.

${ }^{5}$ Blandford, R. D., Q. J. R. Astron. Soc. 31, 305 (1990).

${ }^{6}$ Blandford, R. D. and Narayan, R., Astrophys. J. 310, 568 (1986).

${ }^{7}$ Castrigiano, D. and Hayes, S., Catastrophe Theory (Westview, Bolder, 2004).

${ }^{8}$ Dalal, N. and Rabin, J. M., J. Math. Phys. 42, 1818 (2001).

${ }^{9}$ Golubitsky, M. and Guillemin, V., Stable Mappings and Their Singularities (Springer, Berlin, 1973).
} 
${ }^{10}$ Majthay, A., Foundations of Catastrophe Theory (Pitman, Boston, 1985).

${ }^{11}$ Petters, A. O., J. Math. Phys. 33, 3555 (1993).

${ }^{12}$ Petters, A. O., Levine, H., and Wambsganss, J., Singularity Theory and Gravitational Lensing (Birkhäuser, Boston, 2001).

${ }^{13}$ Schneider, P., Ehlers, J., and Falco, E., Gravitational Lenses (Springer, Berlin, 1992).

${ }^{14}$ Schneider, P. and Weiss, A., Astron. Astrophys. 260, 1 (1992).

${ }^{15}$ Zakharov, A., Astron. Astrophys. 293, 1 (1995). 